\title{
Metastasin leads to poor prognosis of hepatocellular carcinoma through partly inducing EMT
}

\author{
${\text { XIN } \text { ZHENG }^{1 *}, \text { XIAOHONG GAI }}^{1 *}$, ZHENHUA WU $^{2}$, QINGGUANG LIU ${ }^{1}$ and YINGMIN YAO ${ }^{1}$ \\ ${ }^{1}$ Department of Hepatobiliary Surgery, The First Affiliated Hospital of Xi'an Jiaotong University, Xi'an, Shaanxi 710061; \\ ${ }^{2}$ Department of Thoracic Surgery, Xinjiang Tumor Hospital, Urumqi, Xinjiang 830002, P.R. China
}

Received December 19, 2012; Accepted January 18, 2013

DOI: $10.3892 /$ or.2013.2341

\begin{abstract}
Hepatocellular carcinoma (HCC) is a common malignant cancer worldwide characterized by high metastatic potential and poor prognosis following radical resection. Metastasin is a $\mathrm{Ca}^{2+}$-binding protein associated with tumor metastasis. However, the expression and function of metastasin remain unknown. In the present study, we found that the expression of metastasin was upregulated in HCC tissues and positively correlated with poor prognosis following radical resection. Ectopic expression of metastasin in vitro induced typical epithelial-mesenchymal transition (EMT) in Hep3B cells including higher capacity of both migration and invasion, increased expression of both Vimentin and $\mathrm{N}$-cadherin and decreased expression of E-cadherin. Knockdown of metastasin produced the opposite results in $\mathrm{MHCC} 97 \mathrm{H}$ cells, which indicates that metastasin promotes HCC progression via induction of EMT. SNAI1 expression was upregulated by enforced expression of metastasin and, consequently, suppressing upregulation of SNAI1 secondary to metastasin overexpression abolished EMT. Collectively, the present results suggest that metastasin leads to HCC EMT partly through upregulating SNAI1 and contributes to poor prognosis following radical liver resection.
\end{abstract}

\section{Introduction}

Hepatocellular carcinoma (HCC) is the most common type of primary liver cancer and is the third leading cause of cancerrelated mortality worldwide (1). High frequency of intrahepatic and extrahepatic metastasis in the early stage contributes to the poor prognosis of $\mathrm{HCC}$ patients, which is also the vital

Correspondence to: Professor Yingmin Yao, Department of Hepatobiliary Surgery, The First Affiliated Hospital of Xi'an Jiaotong University, 277 Yanta West Road, Xi'an, Shaanxi 710061, P.R. China

E-mail: xin.zheng.xjtu@gmail.com

*Contributed equally

Key words: metastasin, prognosis, hepatocellular carcinoma, epithelialmesenchymal transition factor involved in the disappointing survival following curative liver resection (2). Therefore, it is necessary to establish the factors that act as HCC metastatic markers, while playing an important role in the process of metastasis.

Metastasin, also known as S100A4, belongs to the S100 family of $\mathrm{Ca}^{2+}$-binding proteins encoded by the mts1 gene $(3,4)$. Although the relationship between metastasin and tumor metastasis remains unclear, previous investigations in experimental animal models indicated that metastasin is a metastasis factor via enhancing tumor angiogenesis (5), invasion (6) and motility (7). To our knowledge, metastasin expression is diverse in different organs. High metastasin expression has been found in human monocytes, macrophages and polymorphonuclear granulocytes (8), while low level of metastasin protein was detected in the pancreas, colon, thyroid, lung and kidney (9). However, only few investigations have demonstrated the expression and function of metastasin in liver and HCC.

Epithelial mesenchymal transition (EMT) is a program of cancer metastasis which has been characterized as loss of epithelial cell polarity and acquisition of elongated mesenchymal morphology, concomitant with disruption of cell adhesion, increased cell migration, invasion and metastasis (10). In preliminary studies (11-13), EMT was verified to be involved in the cascade of signaling events inducing HCC metastasis $(14,15)$. However, the concrete mechanisms of induction of EMT in HCC remain unclear. Metastasin was found to induce EMT in renal fibrosis and was renamed fibroblastspecific protein (FSP1) (16). In this investigation, we found that metastasin was significantly associated with poor prognosis of HCC and induced EMT through upregulating SNAI1.

\section{Materials and methods}

Materials. The rabbit anti-metastasin primary antibody (SC-292281, 1:200 dilution) for western immunoblotting was purchased from Santa Cruz Biotechnology (Santa Cruz, CA, USA). The rabbit anti-metastasin primary antibody (ZA-0257) and secondary goat anti-rabbit antibody labeled with biotin (ZDR-5306) for immunohistochemistry was obtained from ZSGB-BIO (Beijing, China). The metastasinexpressing plasmid (RC201616) was from OriGene (Rockville, MD, USA). The human siRNAs targeting both metastasin (SC-106781) and SNAI1 (SC-38398) were purchased from Santa Cruz Biotechnology. Other primary antibodies for 
western immunoblotting included rabbit anti-SNAI1 antibody (C15D3, 1:1,000 dilution; Cell Signaling Technology, Danvers, MA, USA), mouse anti-E-cadherin antibody (610181, 1:1,000 dilution; BD Transduction Laboratories, San Jose, CA, USA), rabbit anti-N-cadherin antibody (sc7939, 1:200 dilution; Santa Cruz Biotechnology), and rabbit anti-Vimentin antibody (EPR3776, 1:1,000 dilution; Abcam, Cambridge, MA, USA). Complete Mini Protease Inhibitor Mixture, anti$\beta$-actin primary antibody (A-5316) and anti-mouse secondary antibodies conjugated with HRP (A3673) were from Sigma Chemical Co. (St. Louis, MO, USA). The anti-rabbit secondary antibodies conjugated with HRP (ALI3403) were from Biosource Co. (Carlsbad, CA, USA), and ECL reagents were from Amersham/GE Healthcare (Piscataway, NJ, USA).

Cell culture and transfection. A previous study showed that there was high metastasin expression in MHCC97H cells, while Hep3B cells expressed metastasin slightly (17). Hence, in the present investigation, $\mathrm{MHCC} 97 \mathrm{H}$ cells were selected as the cell model for metastasin knockdown and Hep3B cells were used for metastasin-overexpression study. MHCC $97 \mathrm{H}$ cell lines were obtained from Fudan University of China and were maintained in our lab. MHCC97H cells, with high metastasis potential frequently used as cell models in the investigation of HCC metastasis, were grown here in DMEM supplemented with 10\% FBS. The Hep3B cell line with limited metastasis capacity was obtained from the American Type Culture Collection (ATCC, Manassas, VA, USA) and was cultured in complete MEM medium with 10\% FBS.

Both metastasin expressing plasmid and control plasmid (pCMV6-Entry) were transfected into Hep3B cells with Roche FuGENE ${ }^{\circledR} 6$ Transfection reagent (Indianapolis, IN, USA) and stably expressing clones were selected by G418 at a dose of $300 \mu \mathrm{g} / \mathrm{ml}$ for two weeks. Western immunoblotting and qRT-PCR assay confirmed overexpression of metastasin in the selected stably expressing clones (Fig. 1). Metastasin siRNA and scramble siRNA were transfected into MHCC97H cells using the siPORT ${ }^{\mathrm{TM}} \mathrm{NeoFX}^{\mathrm{TM}}$ Transfection Agent from Applied Biosystems (Carlsbad, CA, USA). Knockdown of metastasin was verified by both western immunoblotting and qRT-PCR assay in MHCC97H cells transfected with metastasin siRNA (Fig. 2). Silencing SNAI1 expression of Hep3B cells transfected with metastasin expressing plasmid was carried out with the siPORT ${ }^{\mathrm{TM}}$ NeoFX ${ }^{\mathrm{TM}}$ Transfection Agent.

HCC tissue specimens and the matched HCC-adjacent normal tissue specimens. A total of 41 patients with $\mathrm{HCC}$ were enrolled in the study between January 2008 and March 2009, and included 29 men and 12 women (median age, 45 years; range, 34-74 years) who had not received preoperative chemotherapy or embolization. After receiving the routine preoperative examination including chest X-ray, abdominal ultrasonography and computed tomography, all patients underwent liver resection, including radical resection for early $\mathrm{HCC}$ and palliative resection for advanced HCC. Tumor tissues and adjacent liver tissues ( $>2-\mathrm{cm}$ distance to the resection margin) were collected and immediately stored in paraformaldehyde for immunohistochemistry. Clinical data were obtained from the medical records including histopathologic Edmonson classification, clinical tumor-node-metastasis (TNM) grading, maximum

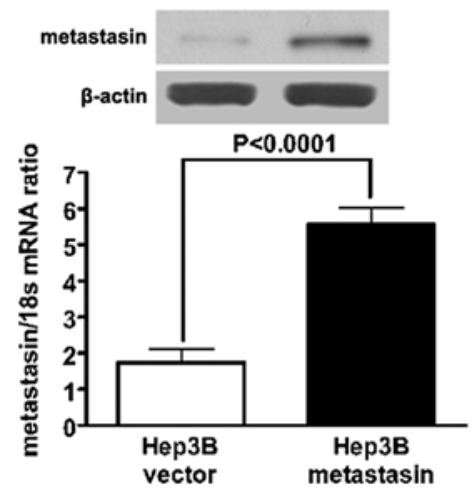

Figure 1. The expression of metastasin was increased after Hep3B cells were stably transfected with metastasin expressing plasmid at the protein and mRNA level.

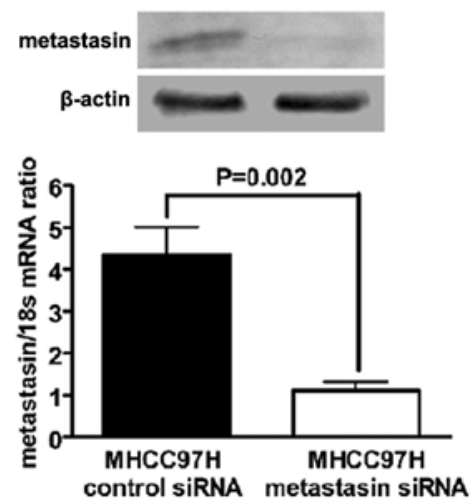

Figure 2. The protein and mRNA expression of metastasin in MHCC97H cells was successfully silenced by siRNA.

tumor diameter, and the tumor-adjacent normal tissues, and were all confirmed by an experienced pathologist who was blinded to clinical information. We also obtained healthy liver tissues from 8 patients without any liver disease. Written informed consent was obtained from all patients. All protocols were approved by the Xi'an Jiaotong University Ethics Committee according to the Helsinki Declaration of 1975.

Immunohistochemistry staining. Briefly, all tissues were placed on glass slides, rehydrated and incubated in $3 \%$ hydrogen peroxide to block the endogenous peroxidase activity. Following trypsinization, sections were blocked by incubation in 3\% bovine serum albumin in PBS. The primary rabbit anti-human metastasin antibody was applied to the slides at a dilution of $1: 150$ and incubated at $4^{\circ} \mathrm{C}$ overnight. The tissues were washed three times with PBS and treated with secondary goat anti-rabbit antibody labeled with biotin at $37^{\circ} \mathrm{C}$ for $30 \mathrm{~min}$. The staining of sections was carried out using HRP-streptavidin conjugates. All sections were visualized with diaminobenzidine and counterstained with hematoxylin. Finally, the sections were dehydrated in alcohol and xylene and mounted onto glass slides.

All slides were detected independently by two experienced pathologists. The staining results were evaluated by an immunohistochemical score combined with the percentage of tumor cells showing specific immunoreactivity. Staining 
intensity was classified into four grades: 0 (none), 1 (weak), 2 (moderate) and 3 (strong). The percentage of positive tumor cells was given with the following grades: $0(<5 \%), 1(6-25 \%)$, $2(26-50 \%), 3(51-75 \%)$ and $4(>75 \%)$. Staining intensity and average percentage of positive tumor cells were assayed in ten independent high-magnification (x400) fields. The total score was calculated by multiplying the staining intensity and the percentage of positive tumor cells. Total score of zero was considered as negative staining. Sections with a total score of 1-4 were defined as the light positive staining, while sections with a score of $>4$ were considered strongly positive staining.

Quantitative real-time reverse transcription polymerase chain reaction ( $q R T-P C R$ ). Total RNA was extracted from HCC cell lines including Hep3B and MHCC97H using the Qiagen RNeasy kit (Valencia, CA, USA). cDNA synthesis was carried out using the High Capacity cDNA Reverse Transcription kit (Applied Biosystems) to transcribe $2 \mu \mathrm{g}$ of total RNA. ABI TaqMan assays were used for qRT-PCR assay in an ABI 7300 system. The ABI TaqMan probes used here were: 18s rRNA (Hs99999901_s1), metastasin (Hs00243201_m1), SNAI1 (Hs 00195591_m1), E-cadherin (Hs 00170423_m1), N-cadherin (Hs 00983062_m1) and Vimentin (Hs 00185584_m1). All TaqMan probes were obtained from Applied Biosystems. The mRNA levels of metastasin, SNAI1, E-cadherin, N-cadherin and Vimentin were normalized to 18s rRNA mRNA levels in the same samples. Each measurement was performed 3 times.

Migration assay. The wound healing assay was performed as the migration assay. Briefly, HCC cells were seeded onto 6 -well plates and cultured to confluency. Scratch wounds were made with a 1,000- $\mu 1$ pipette tip. The wounds were photographed with a phase-contrast microscope at 0,24 and $48 \mathrm{~h}$. Cell migration was quantitated by measuring the width of the wounds. The experiments were performed with at least six replicates.

Invasion assay. A novel quantitative transwell chamber with the Matrigel gel kit - QCM ${ }^{\text {TM }}$ 96-well Cell Invasion Assay kit from Millipore (Billerica, MA, USA) was used to detect the invasion capacity of tumor cells, according to the manufacturer's protocol. Briefly, $\sim 1 \times 10^{5}$ tumor cells were plated into the upper chamber in serum-free medium and medium with $20 \%$ FBS was added into the lower chamber. After $24 \mathrm{~h}$, cells under the invasion chamber were dislodged completely by the cell detachment solution and added into the lysis buffer/dye solution mixture. The results were obtained with a fluorescence plate reader using a 480/520-nm filter set. The experiments were performed with at least six replicates.

Statistical analysis. Differences between groups were compared with the Fisher's exact test or the Mann-Whitney test. Differences between the Kaplan-Meier curves of HCC patients with high and low metastasin expression in the HCC tissue in comparison with the adjacent benign tissue were analyzed with the log-rank test.

\section{Results}

Metastasin is overexpressed in HCC tissues and is associated with poor prognosis. The majority of positive staining
Table I. Correlation between clinicopathological characteristics and metastasin expression in HCC tissues.

\begin{tabular}{|c|c|c|c|c|}
\hline \multirow{4}{*}{ Gender } & \multicolumn{2}{|c|}{$\begin{array}{l}\text { Metastasin } \\
\text { expression }\end{array}$} & \multirow[b]{2}{*}{ Percentage } & \multirow[b]{2}{*}{$\mathrm{P}$-value } \\
\hline & Positive & Negative & & \\
\hline & & & & \\
\hline & 20 & 9 & 60 & 0.514 \\
\hline Female & 7 & 5 & 58.3 & \\
\hline \multicolumn{5}{|c|}{ Age (years) } \\
\hline$\leq 45$ & 14 & 8 & 63.6 & 0.747 \\
\hline$>45$ & 13 & 6 & 68.4 & \\
\hline \multicolumn{5}{|c|}{ HBV infection } \\
\hline Yes & 18 & 10 & 64.3 & 0.756 \\
\hline No & 9 & 4 & 69.2 & \\
\hline \multicolumn{5}{|c|}{ Liver cirrhosis } \\
\hline Yes & 23 & 11 & 67.6 & 0.594 \\
\hline No & 4 & 3 & 57.1 & \\
\hline \multicolumn{5}{|c|}{$\operatorname{AFP}(\mathrm{ng} / \mathrm{ml})$} \\
\hline$\leq 400$ & 12 & 8 & 60.0 & 0.440 \\
\hline$>400$ & 15 & 6 & 71.4 & \\
\hline \multicolumn{5}{|c|}{ Intrahepatic metastasis } \\
\hline Yes & 19 & 5 & 79.2 & 0.033 \\
\hline No & 8 & 9 & 47.1 & \\
\hline \multicolumn{5}{|c|}{ Tumor size $(\mathrm{cm})$} \\
\hline$\leq 5$ & 14 & 10 & 58.3 & 0.228 \\
\hline$>5$ & 13 & 4 & 76.5 & \\
\hline \multicolumn{5}{|c|}{ Portal vein invasion } \\
\hline Yes & 19 & 3 & 86.4 & 0.001 \\
\hline No & 8 & 11 & 42.1 & \\
\hline \multicolumn{5}{|c|}{ Edmonson classification } \\
\hline $\mathrm{I}+\mathrm{II}$ & 17 & 13 & 56.7 & 0.040 \\
\hline III+IV & 10 & 1 & 90.9 & \\
\hline \multicolumn{5}{|c|}{ TNM staging } \\
\hline $\mathrm{I}+\mathrm{II}$ & 12 & 11 & 52.2 & 0.037 \\
\hline III+IV & 15 & 3 & 83.3 & \\
\hline
\end{tabular}

cells showed diffuse cytoplasmic staining of metastasin, while nuclear staining was found in a few cells (Fig. 3A). In 41 pairs of $\mathrm{HCC}$ tissues and adjacent liver tissues, there were $27(65.9 \%)$ positive metastasin staining HCC tissues compared to $8(19.5 \%)$ positive staining adjacent liver tissues (Fig. 3A and $\mathrm{B})$. The staining of metastasin in the 8 healthy liver tissues was negative (Fig. 3C). These results clearly indicate that metastasin was overexpressed in HCC tissues $(\mathrm{P}<0.001)$. After analyzing the relationship between metastasin expression in HCC tissues and clinicopathological characteristics (Table I), we found that positive metastasin expression was significantly related to intrahepatic metastases $(\mathrm{P}=0.033)$, portal vein invasion $(\mathrm{P}=0.001)$, advanced TNM staging $(\mathrm{P}=0.037)$ and high Edmonson classification $(\mathrm{P}=0.04)$, suggesting that metastasin expression in HCC tissues contributes to HCC metastasis. 
A

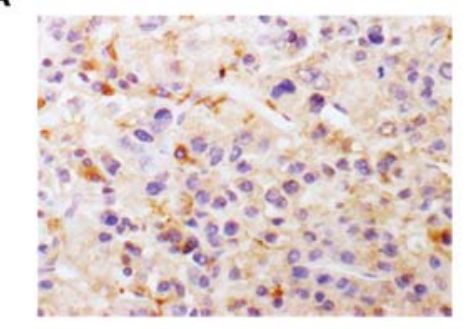

C

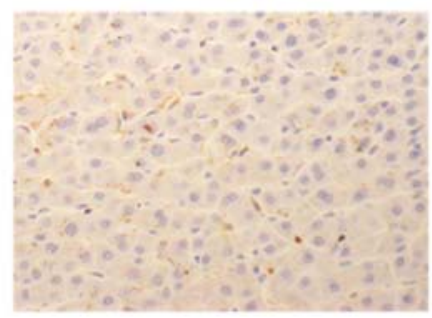

D

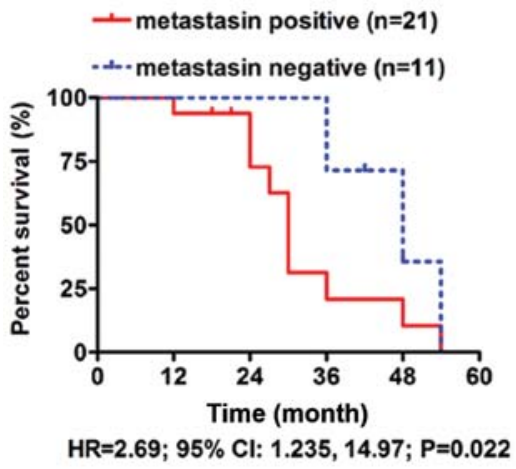

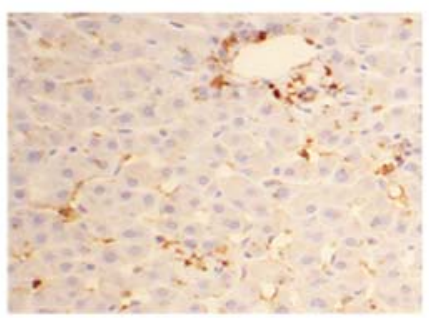

E

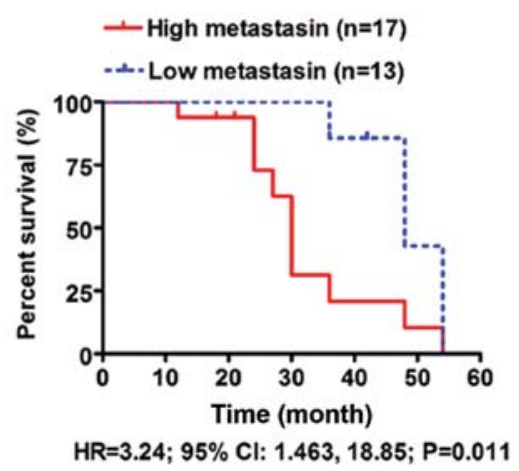

Figure 3. Metastasin is frequently overexpressed in HCC tissues compared to adjacent normal tissues and is associated with poor survival following liver resection. (A) Immunohistochemistry staining of metastasin in HCC tissue. (B) Immunohistochemistry staining of metastasin in tumor-adjacent normal liver tissue. (C) Immunohistochemistry staining of metastasin in normal liver tissue. (D) Differences between the Kaplan-Meier curves of HCC patients in the metastasin positive group and those in the metastasin negative group. (E) Differences between the Kaplan-Meier curves of HCC patients in the high metastasin group and those in the low metastasin group. Original magnification of immunohistochemistry, x200.

We obtained follow-up information from 32 of the $41 \mathrm{HCC}$ cases $(78 \%)$. The median duration of follow-up was 24 months. The 3-year survival rate of the metastasin positive group was $20.8 \%$ compared to $71.4 \%$ in the metastasin negative group. As compared by the Kaplan-Meier survival curve, HCCs with metastasin expression were found to have poorer survival $(\mathrm{HR}=2.69$; 95\% CI: 1.235, 14.97; $\mathrm{P}=0.022$ ) (Fig. 3D). We also compared the survival curves between the high metastasin group, in which HCC tissues expressed more metastasin than adjacent normal tissues, and in the low metastasin group. The 3 -year survival rate of the high metastasin group was found to be $20.8 \%$ compared to $85.7 \%$ in the low metastasin group. The high metastasin group was found to have clearly worse overall survival than the low metastasin group (HR=3.24; 95\% CI: $1.463,18.85 ; \mathrm{P}=0.011$ ) (Fig. 3E). These data showed that metastasin expression in HCC tissues was markedly associated with poor prognosis.

Ectopic expression of metastasin in Hep3B cells promotes cell migration and invasion, and induces EMT. Ectopic expression of metastasin was confirmed by both qRT-PCR and western immunoblotting (Fig. 1). To investigate the effect of metastasin on HCC migration, wound healing assay was carried out and showed that the migration rate of Hep3B cells stably transfected with metastasin expressing plasmid (Hep3B metastasin cells) was significantly faster than that of Hep3B cells transfected with vector plasmid (Hep3B vector cells) at both 24 and $48 \mathrm{~h}$ after scratching (both P-values were 0.002; Fig. 4A). Similar results were obtained from the quantitative invasion assay, which showed that the invasion ability of Hep3B cells was greatly increased by ectopic expression of metastasin ( $\mathrm{P}=0.001$; Fig. 4B).

To investigate whether metastasin promotes migration and invasion by inducing EMT, we examined expression of EMT markers of Hep3B cells following overexpression of metastasin. SNAI1, a well-known EMT inducer, was found to be upregulated at both the mRNA and the protein level. qRT-PCR assay revealed that E-cadherin mRNA expression of Hep3B cells was decreased by $\sim 60 \%$ by ectopic expression of metastasin (Fig. 4C). The protein expression of E-cadherin was verified to be suppressed in Hep3B metastasin cells by western immunoblotting (Fig. 4D). On the other hand, as shown in Fig. 4D, the expression of both $\mathrm{N}$-cadherin and Vimentin, which are considered mesenchymal markers, was found enhanced at the mRNA and protein levels by metastasin overexpression. Collectively, the data presented here indicate 
A
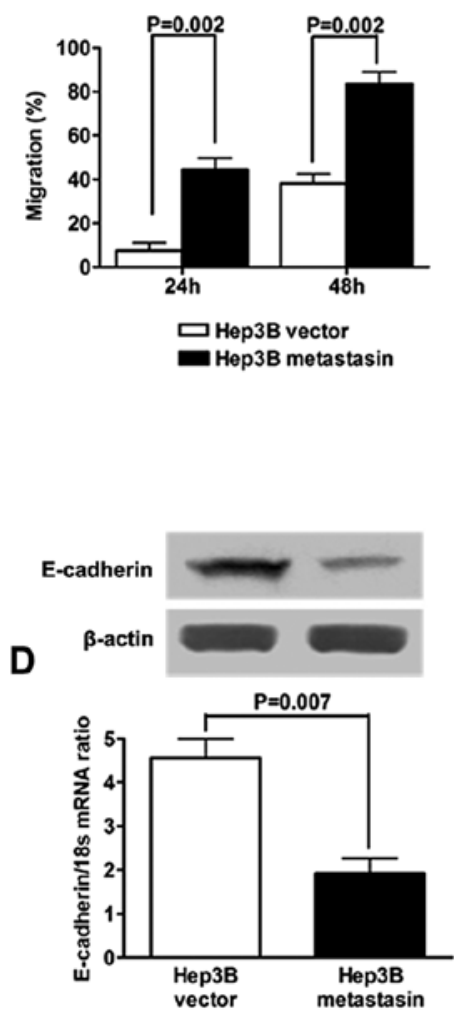

B
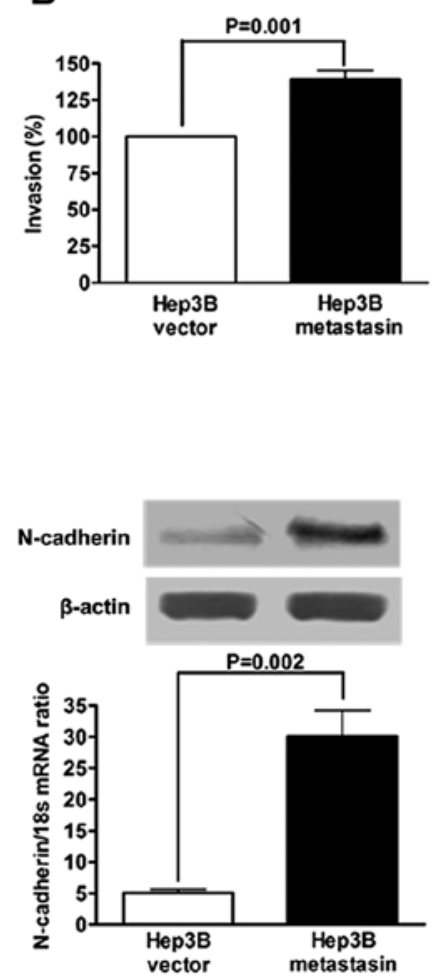
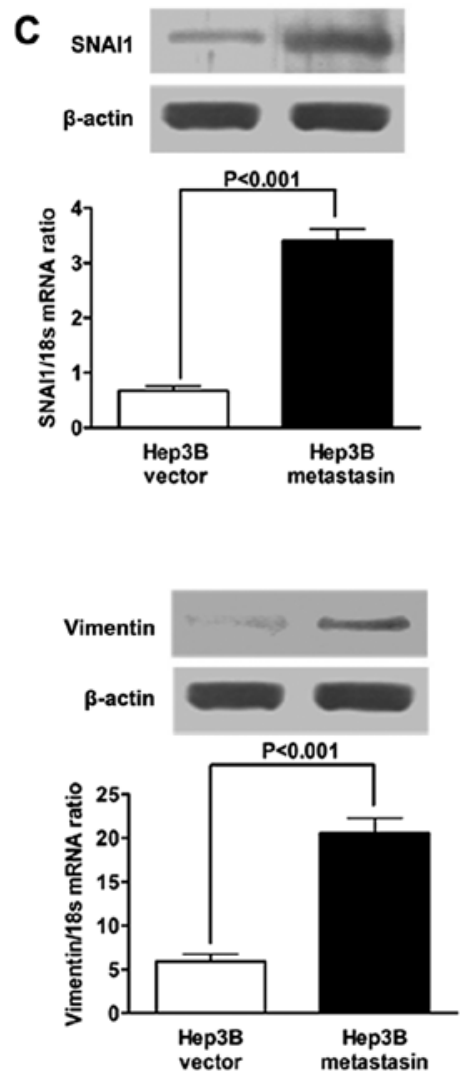

Figure 4. Forced expression of metastasin promotes both migration and invasion and induces EMT phenotype of Hep3B cells. (A) Migration of Hep3B cells was significantly increased by metastasin overexpression as assessed by the wound healing assay. (B) Forced expression of metastasin clearly enhanced the invasion capacity of Hep3B cells. (C) SNAI1 expression of Hep3B cells was found upregulated $>3$-fold following metastasin overexpression by both qRT-PCR and western immunoblotting. (D) qRT-PCR and western immunoblotting showed that ectopic expression of metastasin decreased E-cadherin expression and markedly increased the expression of two types of mesenchymal markers, $\mathrm{N}$-cadherin and Vimentin.

that metastasin promotes migration and invasion of Hep3B cells via induction of EMT.

Knockdown of metastasin in MHCC97H cells suppresses cell migration and invasion and reverses EMT. To further confirm the observation regarding metastasin overexpression, we silenced metastasin expression of $\mathrm{MHCC} 97 \mathrm{H}$ cells by transfection of metastasin siRNA (Fig. 2). As shown in Fig. 5A, wound healing assay showed that knockdown of metastasin significantly suppressed mobility of $\mathrm{MHCC} 97 \mathrm{H}$ cells $(24 \mathrm{~h}$ after scratching, $\mathrm{P}=0.032 ; 48 \mathrm{~h}$ after scratching, $\mathrm{P}=0.011)$. Accordingly, the invasion ability of $\mathrm{MHCC} 97 \mathrm{H}$ cells was markedly attenuated by silencing metastasin $(\mathrm{P}=0.002$; Fig. 5B).

Next, we tested if knockdown of metastasin downregulated SNAI1 expression and reverted EMT in $\mathrm{MHCC} 97 \mathrm{H}$ cells. SNAI1 expression was significantly decreased by silencing metastasin at both the protein and the mRNA level, as assessed by western immunoblotting and qRT-PCR, respectively (Fig. 5C). Further examination for the expression of EMT markers showed that knockdown of metastasin promoted E-cadherin expression and suppressed the expression of N-cadherin and Vimentin (Fig. 5D). The complete opposite results obtained here further confirm the hypothesis that metastasin induces EMT in HCC cells and then promotes cell migration and invasion.
Suppression of SNAII blocks EMT induced by metastasin. To investigate the role of SNAI1 in metastasin-induced EMT, we silenced SNAI1 upregulated by metastasin overexpression and re-evaluated EMT phenotype of Hep3B metastasin cells. qRT-PCR and western immunoblotting confirmed that SNAI1 expression of Hep3B cells was knocked down effectively by siRNA (Fig. 6A). E-cadherin expression was increased significantly, while the expression of both $\mathrm{N}$-cadherin and Vimentin were decreased (Fig. 6B). The migration and invasion of Hep3B metastasin cells were both significantly inhibited (Fig. 6C and D). These data showed that EMT phenotype of Hep3B cells induced by metastasin was reversed following SNAI1 knockdown.

\section{Discussion}

HCC is one of the most common malignant tumors worldwide with increasing incidence rates. Radical hepatic resection at the early stage is the most important and common curative treatment for HCC. However, due to the lack of typical symptoms at the early stage and high metastatic capacity, the majority of HCC patients at present have no opportunity to receive the curative resection. To date, metastasis of $\mathrm{HCC}$ remains largely incurable due to its systemic characteristics and the resistance to the existing therapeutic agents. Therefore, it is critical to explore the mechanism underlying HCC metas- 

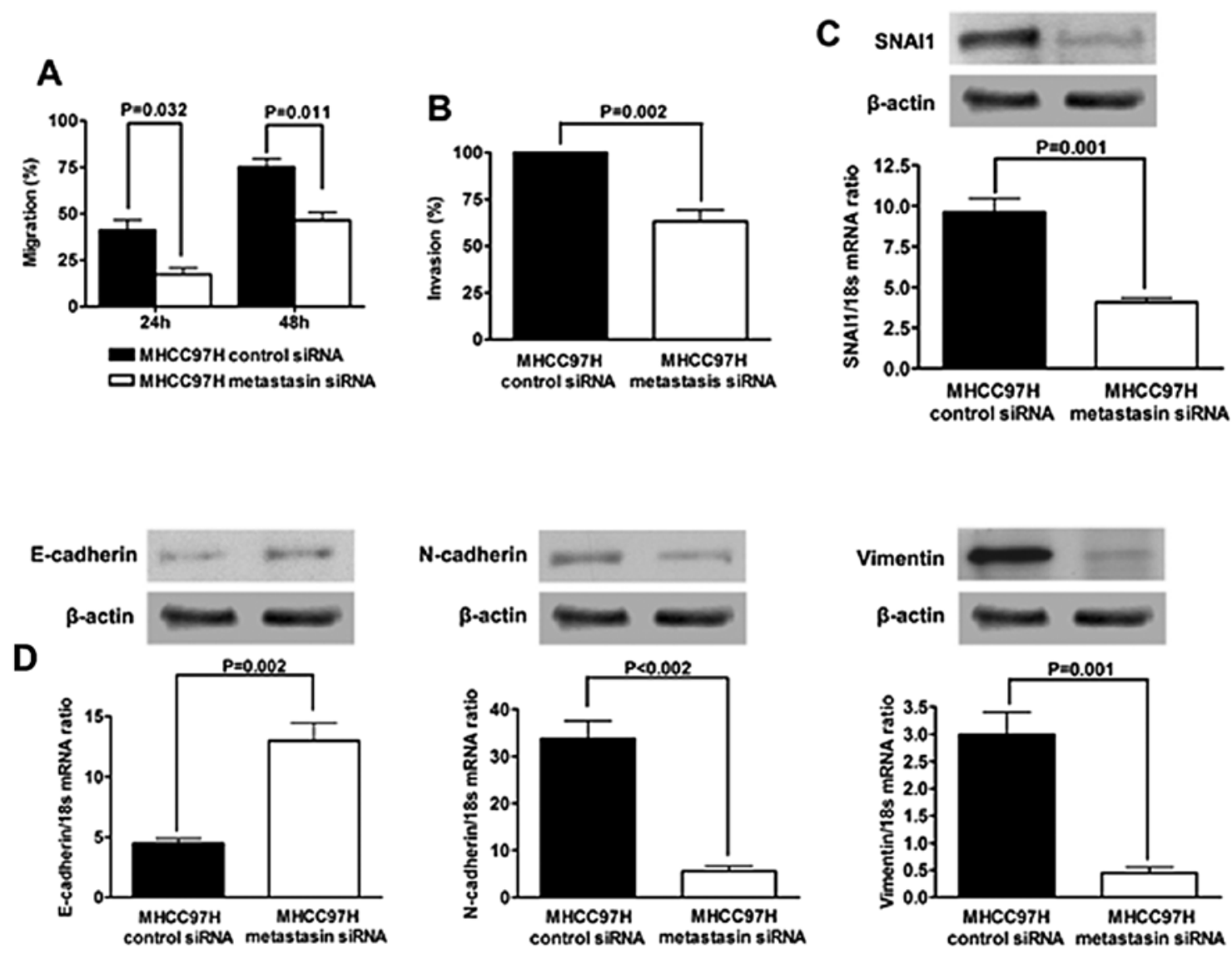

Figure 5. Knockdown of metastasin inhibits both migration and invasion ability and reverses EMT phenotype of MHCC97H cells. (A) Migration of Hep3B cells was found significantly suppressed by silencing metastasin, as assessed by the wound healing assay. (B) Knockdown of metastasin clearly inhibited the invasion potential of MHCC97H cells. (C) SNAI1 expression of MHCC97H cells was found evidently decreased by knockdown of metastasin by both qRTPCR and western immunoblotting. (D) Silencing metastasin increased E-cadherin expression and downregulated the expression of N-cadherin and Vimentin significantly at both the mRNA and the protein level.

tasis and to develop effective clinical prognostic biomarkers and therapeutic agents.

The metastasin protein encoded by the human mts1 gene located at position 1q21 on chromosome 1 is a type of polypeptide of 101 amino acids with the molecular mass of $11.5 \mathrm{kDa}$. Previous studies revealed that the transcription of metastasin is controlled by regulatory elements in its first intron via binding with several transcription factors, such as I- $\kappa \mathrm{B}(18,19)$. Epigenetic investigations also found metastasin transcription was strongly affected by the methylation of both its enhancer and silencer elements, which could contribute to its aberrant expression in diverse types of human cancer (20-22). Clinical research on breast cancer patients with regional invasion but operable stage I and II, reported by Rudland et al (23), showed that during the 19-year follow-up period, the median survival of the metastasin negative group was 228 months vs. 47 months in the metastasin positive group. A similar result was reported by Lee et al (24) showing that metastasin was related with poor outcome of T1N0M0 breast cancer and could be a biomarker of early metastasis of breast cancer. Aside from the investigations on breast cancer, numerous studies of different types of human cancer, including colon cancer (25), gastric cancer $(26,27)$, pancreatic ductal carcinoma (28), renal carcinoma (29), found that metastasin expression was significantly overexpressed in malignant tissues and was associated with poor survival. To our knowledge, there are few reports about the expression and function of metastasin on HCC. In this study, we found metastasin was aberrantly upregulated in HCC tissues and was associated with poor outcome following liver resection, which indicates metastasin could be a potential predictive marker and therapy target for HCC.

In the present study we initially investigated the mechanism by which metastasin leads to poor survival of HCC. In general, metastasin has no enzymatic activity and works via binding and interacting with other proteins, including p53, P37, S100A1, CCN3 (30,31). Numerous in vivo experiments showed that metastasin promotes tumor metastasis in mouse models. Ambartsumian et al (32) verified that metastasin leads to higher metastatic capacity of mammary tumor via transgenic mouse overexpressing metastasin in mammary epithelium. The knockdown in vivo experiments on lung carcinoma and osteosarcoma also showed silencing metastasin suppressed the metastatic potential of tumor cells $(33,34)$. Although several investigations showed overexpression of metastasin induced downregulation of E-cadherin and upregulation of MMPs $(6,35,36)$, the underlying mechanism by which metastasin promotes tumor metastasis has yet to be fully elucidated. Here, we found ectopic expression of metastasin in Hep3B cells increased cell mobility and invasion. SNAI1, a renowned EMT inducer, was also found 


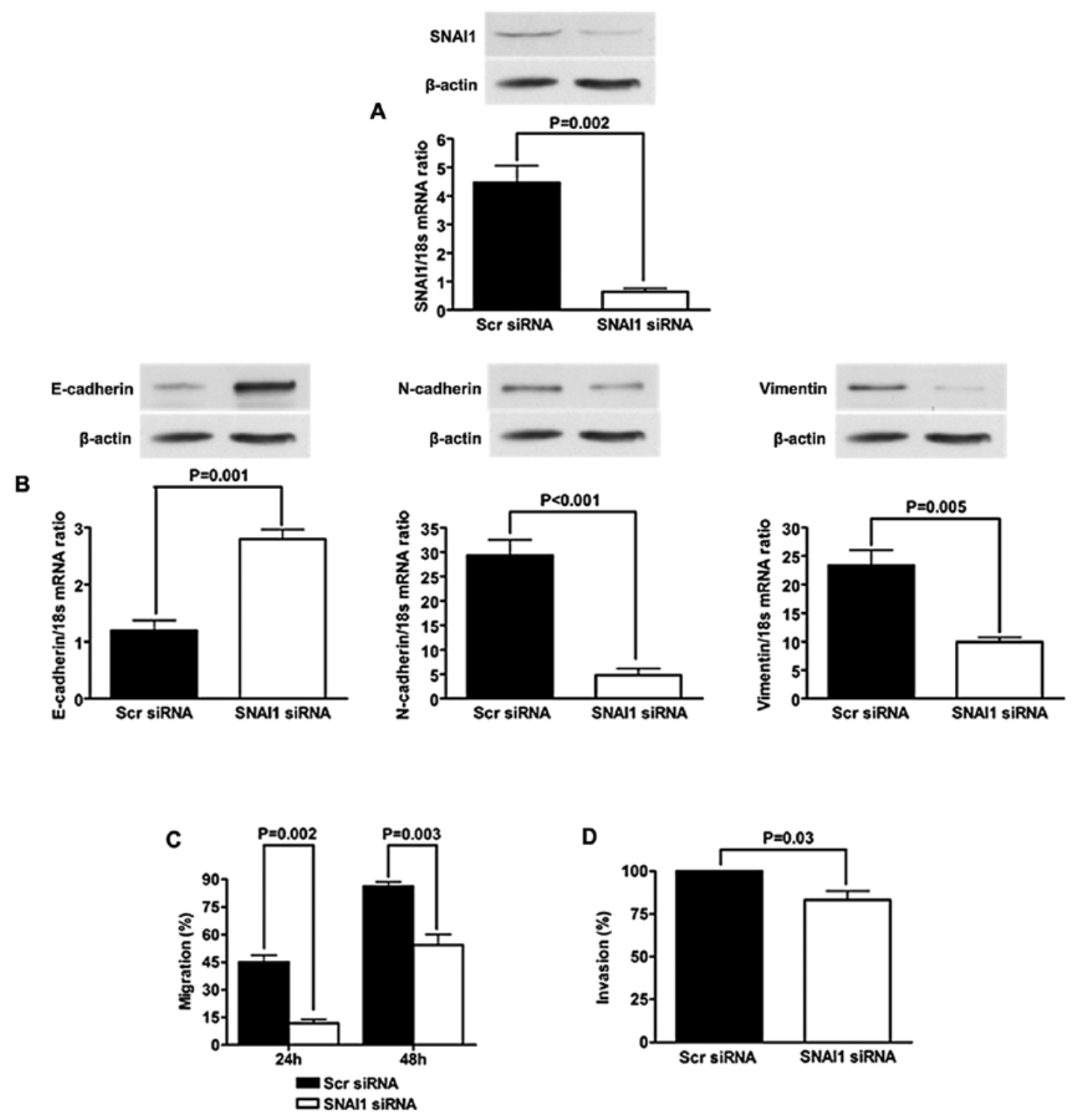

Figure 6. Suppression of SNAI1 blocks EMT induced by metastasin. (A) SNAI1 upregulation by overexpression of metastasin was abolished entirely by transfecting SNAI1 siRNA into Hep3B cells one day prior to transfecting metastasin expressing plasmid. (B) Suppression of SNAI1 clearly increased E-cadherin expression and decreased the expression of both $\mathrm{N}$-cadherin and Vimentin in Hep3B metastasin cells, as assessed by qRT-PCR and western immunoblotting. (C) Cell migration of Hep3B metastasin cells was significantly inhibited by suppression of SNAI1, as assessed by the wound healing assay. (D) The invasion capacity of Hep3B metastasin cells was inhibited by suppression of SNAI1.

upregulated by metastasin overexpression, suggesting that metastasin induced EMT in Hep3B cells. Further examination showing E-cadherin was downregulated and both N-cadherin and Vimentin were upregulated confirms the EMT phenotype of Hep3B cells induced by ectopic expression of metastasin. Conversely, knockdown of metastasin in MHCC97H cells led to the opposite results, which further verifies that metastasin induces EMT phenotype of HCC cells through upregulating SNAI1. Although metastasin was found to enhance SNAI1 expression in both Hep3B and $\mathrm{MHCC} 97 \mathrm{H}$ cells, there is no evidence to support that metastasin could regulate the transcription of SNAI1 directly. Our group sought to find the metastasin-binding site of SNAI1 promoter by carrying out EMSA (unpublished data) and did not get any positive results, which indicates that metastasin could upregulate SNAI1 in indirect ways. To clarify the role of SNAI1 upregulated on metastasin-driven EMT, we successfully inhibited the upregulation of SNAI1 after stably overexpressing metastasin in Hep3B cells by SNAI1 siRNA, and found E-cadherin expression was increased and the expression of both $\mathrm{N}$-cadherin and Vimentin were clearly decreased. Consistently, both cell mobility and invasion were inhibited significantly. Consequently, these data indicate EMT induced by metastasin is reversed by suppressing SNAI1 in Hep3B cells and SNAI1 is involved in the intracellular signal cascade of metastasin-induced EMT.

In summary, this investigation shows metastasin is overexpressed in HCC and is markedly related with poor survival. Metastasin induces EMT phenotype of HCC via upregulating SNAI1, which could explain initially how metastasin leads to poor prognosis of HCC. Therefore, metastasin is a potential clinical biomarker and target for anti-HCC therapy, and further investigation is required to elucidate the concrete mechanism by which metastasin promotes HCC progression. 


\section{Acknowledgements}

This study was supported by grants from the National Natural Science Foundation of China to Y.Y. (81071897/H1612) and to Q.L. (81271645/H1617 and 81072052/H1617).

\section{References}

1. Yang JD and Roberts LR: Hepatocellular carcinoma: a global view. Nat Rev Gastroenterol Hepatol 7: 448-458, 2010.

2. Ma W, Wong CC, Tung EK, Wong CM and Ng IO: RhoE is frequently downregulated in HCC and suppresses HCC invasion through antagonizing the Rho/ROCK/MYPT pathway. Hepatology 57: 152-161, 2012.

3. Berge $\mathrm{G}$ and Maelandsmo GM: Evaluation of potential interactions between the metastasis-associated protein S100A4 and the tumor suppressor protein p53. Amino Acids 41: 863-873, 2011.

4. Mishra SK, Siddique HR and Saleem M: S100A4 calciumbinding protein is key player in tumor progression and metastasis: preclinical and clinical evidence. Cancer Metastasis Rev 31: 163-172, 2012.

5. Ambartsumian N, Klingelhofer J, Grigorian M, et al: The metastasis-associated Mts1(S100A4) protein could act as an angiogenic factor. Oncogene 20: 4685-4695, 2001.

6. Saleem M, Kweon MH, Johnson JJ, et al: S100A4 accelerates tumorigenesis and invasion of human prostate cancer through the transcriptional regulation of matrix metalloproteinase 9. Proc Natl Acad Sci USA 103: 14825-14830, 2006.

7. Stein U, Arlt F, Walther W, et al: The metastasis-associated gene S100A4 is a novel target of beta-catenin/T-cell factor signaling in colon cancer. Gastroenterology 131: 1486-1500, 2006.

8. Takenaga K, Nakanishi H, Wada K, et al: Increased expression of S100A4, a metastasis-associated gene, in human colorectal adenocarcinomas. Clin Cancer Res 3: 2309-2316, 1997.

9. Ilg EC, Schafer BW and Heizmann CW: Expression pattern of S100 calcium-binding proteins in human tumors. Int J Cancer 68: 325-332, 1996.

10. Hanahan D and Weinberg RA: Hallmarks of cancer: the next generation. Cell 144: 646-674, 2011.

11. Hao H, Liu J, Liu G, et al: Depletion of GRIM-19 accelerates hepatocellular carcinoma invasion via inducing EMT and loss of contact inhibition. J Cell Physiol 227: 1212-1219, 2012.

12. Zhu K, Dai Z, Pan Q, et al: Metadherin promotes hepatocellular carcinoma metastasis through induction of epithelial-mesenchymal transition. Clin Cancer Res 17: 7294-7302, 2011.

13. Wang J, Chen L, Li Y and Guan XY: Overexpression of cathepsin $\mathrm{Z}$ contributes to tumor metastasis by inducing epithelial-mesenchymal transition in hepatocellular carcinoma. PLoS One 6: e24967, 2011.

14. Nagai T, Arao T, Furuta K, et al: Sorafenib inhibits the hepatocyte growth factor-mediated epithelial mesenchymal transition in hepatocellular carcinoma. Mol Cancer Ther 10: 169-177, 2011.

15. Xia L, Huang W, Tian D, et al: Overexpression of forkhead box $\mathrm{C} 1$ promotes tumor metastasis and indicates poor prognosis in hepatocellular carcinoma. Hepatology 57: 610-624, 2013.

16. Strutz F, Okada H, Lo CW, et al: Identification and characterization of a fibroblast marker: FSP1. J Cell Biol 130: 393-405, 1995.

17. Cui JF, Liu YK, Zhang LJ, et al: Identification of metastasis candidate proteins among HCC cell lines by comparative proteome and biological function analysis of S100A4 in metastasis in vitro. Proteomics 6: 5953-5961, 2006.
18. Tulchinsky EM, Georgiev GP and Lukanidin EM: Novel AP-1 binding site created by DNA-methylation. Oncogene 12: 1737-1745, 1996.

19. Cohn MA, Hjelmso I, Wu LC, Guldberg P, Lukanidin EM and Tulchinsky EM: Characterization of Sp1, AP-1, CBF and KRC binding sites and minisatellite DNA as functional elements of the metastasis-associated mts1/S100A4 gene intronic enhancer. Nucleic Acids Res 29: 3335-3346, 2001.

20. Li Y, Liu ZL, Zhang KL, et al: Methylation-associated silencing of S100A4 expression in human epidermal cancers. Exp Dermatol 18: 842-848, 2009.

21. Xie R, Loose DS, Shipley GL, Xie S, Bassett RL Jr and Broaddus RR: Hypomethylation-induced expression of S100A4 in endometrial carcinoma. Mod Pathol 20: 1045-1054, 2007.

22. Rehman I, Goodarzi A, Cross SS, et al: DNA methylation and immunohistochemical analysis of the S100A4 calcium binding protein in human prostate cancer. Prostate 67: 341-347, 2007.

23. Rudland PS, Platt-Higgins A, Renshaw C, et al: Prognostic significance of the metastasis-inducing protein S100A4 (p9Ka) in human breast cancer. Cancer Res 60: 1595-1603, 2000.

24. Lee WY, Su WC, Lin PW, Guo HR, Chang TW and Chen HH: Expression of S100A4 and Met: potential predictors for metastasis and survival in early-stage breast cancer. Oncology 66: 429-438, 2004

25. Wang HY, Zhang JY, Cui JT, et al: Expression status of S100A14 and S100A4 correlates with metastatic potential and clinical outcome in colorectal cancer after surgery. Oncol Rep 23: 45-52, 2010.

26. Wang YY, Ye ZY, Zhao ZS, Tao HQ and Chu YQ: High-level expression of S100A4 correlates with lymph node metastasis and poor prognosis in patients with gastric cancer. Ann Surg Oncol 17: 89-97, 2010.

27. Yonemura Y, Endou Y, Kimura K, et al: Inverse expression of S100A4 and E-cadherin is associated with metastatic potential in gastric cancer. Clin Cancer Res 6: 4234-4242, 2000.

28. Ikenaga N, Ohuchida K, Mizumoto K, et al: S100A4 mRNA is a diagnostic and prognostic marker in pancreatic carcinoma. J Gastrointest Surg 13: 1852-1858, 2009.

29. Bandiera A, Melloni G, Freschi M, et al: Prognostic factors and analysis of S100a4 protein in resected pulmonary metastases from renal cell carcinoma. World J Surg 33: 1414-1420, 2009.

30. Tarabykina S, Griffiths TR, Tulchinsky E, Mellon JK, Bronstein IB and Kriajevska M: Metastasis-associated protein S100A4: spotlight on its role in cell migration. Curr Cancer Drug Targets 7: 217-228, 2007.

31. Santamaria-Kisiel L, Rintala-Dempsey AC and Shaw GS: Calcium-dependent and -independent interactions of the S100 protein family. Biochem J 396: 201-214, 2006.

32. Ambartsumian NS, Grigorian MS, Larsen IF, et al: Metastasis of mammary carcinomas in GRS/A hybrid mice transgenic for the mts1 gene. Oncogene 13: 1621-1630, 1996.

33. Maelandsmo GM, Hovig E, Skrede M, et al: Reversal of the in vivo metastatic phenotype of human tumor cells by an anti-CAPL (mts1) ribozyme. Cancer Res 56: 5490-5498, 1996.

34. Takenaga K, Nakamura $\mathrm{Y}$ and Sakiyama S: Expression of antisense RNA to S100A4 gene encoding an S100-related calcium-binding protein suppresses metastatic potential of highmetastatic Lewis lung carcinoma cells. Oncogene 14: 331-337, 1997.

35. Moriyama-Kita M, Endo Y, Yonemura Y, et al: S100A4 regulates E-cadherin expression in oral squamous cell carcinoma. Cancer Lett 230: 211-218, 2005.

36. Schmidt-Hansen B, Ornas D, Grigorian M, et al: Extracellular S100A4(mts1) stimulates invasive growth of mouse endothelial cells and modulates MMP-13 matrix metalloproteinase activity. Oncogene 23: 5487-5495, 2004. 